\title{
Projeto de microssensor de fluxo de massa para medição de velocidade de fluidos
}

\author{
Deivity C. Santos* Alípio M. Barbosa** \\ Michel F. S. Moreira ${ }^{* *}$ \\ * Departamento de Engenharia Elétrica, Universidade Federal de São \\ João del-Rei, MG, (e-mail: dsantos1995@hotmail.com). \\ ** Grupo de Modelagem, Automação e Controle, Centro Universitário \\ Newton Paiva, MG, (e-mails: alipio.barbosa@newtonpaiva.br, \\ michel.moreira@newtonpaiva.br)
}

\begin{abstract}
In this paper, the main characteristics and steps for design and simulation of a thermal mass flow microsensor of calorimetric type are described, aiming to measure flow rate and fluid velocity. The device was designed computationally and its applicability evaluated based on the simulation data. The results leaded to conclude that flows speed between 0 and $14 \mathrm{~m} / \mathrm{s}$ can be measured using the proposed sensor.

Resumo: Neste trabalho são descritas as principais características e etapas de projeto e simulação de um microssensor de fluxo de massa do tipo calorimétrico, tendo como objetivo aferir vazão e velocidade de fluidos. O dispositivo foi projetado computacionalmente e sua aplicabilidade avaliada a partir dos resultados de simulação. Os dados obtidos permitiram concluir que a velocidades entre 0 e $14 \mathrm{~m} / \mathrm{s}$ podem ser mensuradas por meio do aparato proposto.
\end{abstract}

Keywords: System simulation; instrumentation; microsensor; MEMS.

Palavras-chaves: Simulação de sistemas; instrumentação; microssensor; MEMS.

\section{INTRODUÇÃO}

Desde os primórdios dos tempos, o homem busca formas de otimizar a execução das tarefas de trabalho. Na chamada Revolução Industrial, com início no século 18, o uso das máquinas substituiu os processos manufaturados e, desde então, significativos avanços tecnológicos são observados em todas as etapas produtivas. Atualmente tal movimento encontra-se em sua quarta fase, a chamada Indústria 4.0, que sugere alto grau de automação e interconectividade dos sistemas industriais (Lu, 2017).

O desenvolvimento das tecnologias trouxe consigo sistemas de produção mais rápidos, flexíveis, confiáveis e robustos, o que também culminou no aumento da complexidade dos mesmos no que tange a operação e gerência de recursos. Essas características geraram uma demanda por uma maior distribuição do processo de tomada de decisão, sem a perda da coordenação entre as partes (Vaidya et al., 2018). Desta forma, recursos da Tecnologia da Informação (TI), da inteligência artificial, big data e cloud computing são cada vez mais difundidos nos ambientes industriais, bem como em aplicações automotivas, médicas e de outras naturezas ( $\mathrm{Lu}, 2017)$.

Outra característica bastante peculiar dessa fase de avanços tecnológicos é a presença de dispositivos de dimensões físicas criticamente reduzidas, possíveis graças às técnicas de manuseio de metais e materiais semicondutores (Elwenspoek, 1999). Os MEMS (Microelectromechanical Systems), por exemplo, são parte de uma tecnologia relativamente recente, estudada sobretudo nas últimas três décadas. Nessa categoria estão inclusos sensores, atuadores e outros mecanismos cujos elementos são da ordem de micrômetros, e o seu funcionamento explora ao máximo propriedades físico-químicas dos materiais utilizados na construção dos mesmos. A aplicação destes é comum em circuitos integrados, sistemas embarcados e nós de redes sensoriais (Maluf, 2002; Haasl e Stemme, 2008).

Os sensores de fluxo de massa vem sendo amplamente explorados e incorporados à tecnologia dos MEMS com as mais diversas finalidades. Desde a área médica (Inoue et al., 2018), passando por sistemas de medição e automação (Melnyk et al., 2018), até instrumentos de detecção de gases (Gerdroodbary et al., 2018). Os sensores do tipo calorimétrico, especificamente, são apropriados para uma grande variedade de propósitos. Todavia, em alguns casos, sua aplicação é limitada pela velocidade máxima que o dispositivo é capaz de mensurar (Djuzhev et al., 2018).

O presente trabalho tem por objetivo demonstrar o princípio de funcionamento, as etapas de desenvolvimento e os resultados da simulação computacional de um microssensor de fluxo de massa, concebido sob a perspectiva da tecnologia MEMS. Buscou-se ampliar a faixa de operação do instrumento, em comparação com outros registros da literatura, por meio da definição da geometria dos elementos e seleção dos materiais utilizados.

Este documento está organizado da seguinte forma: na seção 2 é apresentada a fundamentação teórica para o projeto do sensor, na seção 3 são descritos os procedimentos metodológicos e testes realizados. Na seção 4, os resultados 
obtidos e as análises pertinentes são exibidos e, por fim, na seção 5 são apresentadas as conclusões.

\section{MICROSSENSORES DE FLUXO}

Os microssensores de fluxo de massa térmica são dispositivos destinados à medição de velocidade ou vazão de fluidos, estes são compostos basicamente por um aquecedor e elementos sensíveis à variação de temperatura. Suas dimensões, criticamente reduzidas se comparado aos sensores convencionais, são possíveis graças ao emprego da tecnologia MEMS (Microelectromechanical Systems) e geralmente são fabricados combinando-se metais e materiais semicondutores (Elwenspoek, 1999).

Maluf (2002) define a tecnologia MEMS como um portfólio de técnicas e processos para projetar e criar sistemas que envolvem um ou mais componentes e estruturas microfabricadas. Outra possível classificação para estes dispositivos, ainda mais adequada, é como subsistemas ou componentes, uma vez que estes geralmente estão ligados à execução e/ou controle de uma tarefa bastante específica. Destacam-se como vantagens de se utilizar sensores e atuadores em micro e nanoescala o baixo consumo de energia durante seu funcionamento e a possibilidade de integrá-los a sistemas de pequeno porte, o que propicia que estes sejam naturalmente viáveis no âmbito da eletrônica embarcada (Sazhin, 2013). Nesse sentido, os estudos acerca dos microssensores tem se tornado recorrentes, bem como sua aplicação, que já é uma realidade presente nos mais diversos setores da indústria.

Em suma, microssensores de fluxo de massa térmica podem ser divididos em três grandes categorias: anemômetros, calorimétricos e TOF (time of flight). Esta classificação é dada de acordo com seu princípio de funcionamento, sendo que alguns dispositivos podem pertencer a mais de uma categoria (Elwenspoek, 1999).

Os anemômetros térmicos, também podem ser chamados de anemômetros a fio-quente, hot-chip ou hot-film, são sensores que mensuram o fluxo de determinado fluido baseado na dissipação de calor (Haasl e Stemme, 2008). Esse modelo do transdutor é o primeiro de que se tem registro e seu uso é muito comum em processos onde é necessária a medição de velocidade e vazão de fluidos líquidos e gasosos.

O elemento central do instrumento é um fio eletricamente aquecido que se localiza no centro do canal por onde o fluido percorre, o mesmo é sustentado por bases compostas de materiais com boa isolação térmica (Haasl e Stemme, 2008). Elwenspoek (1999) demonstra duas formas de operação dos anemômetros térmicos: potência constante e temperatura constante. No primeiro caso, a temperatura do aquecedor é a grandeza a ser mensurada e correlacionada ao fluxo. O segundo modo de operação caracteriza-se por manter a temperatura constante, enquanto a potência fornecida para manter essa temperatura é mensurada. O anemômetro térmico é ilustrado na Figura 1.

Sensores baseados nesse princípio de funcionamento podem variar muito suas características construtivas, podendo conter mais elementos aquecedores e apresentar variações nas dimensões e no formato (Elwenspoek, 1999; Sazhin, 2013). Barmpakos et al. (2019) apresentam uma

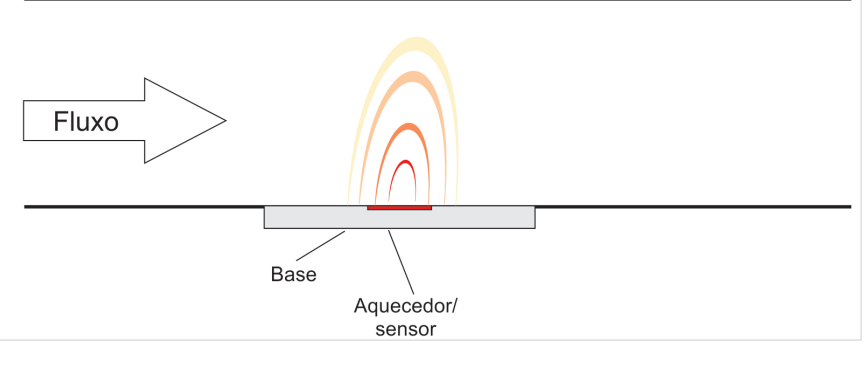

Figura 1. Anemômetro térmico.

alternativa onde os elementos sensores são dispostos radialmente a fim de se estimar também a direção do fluido.

Por via de regra, os sensores calorimétricos são constituídos por elementos sensores, que podem ser termorresistências ou RTDs (Resistence Temperature Dependent), alocados simetricamente em relação a um aquecedor (Figura 2). Os itens são dispostos em uma base feita de material com baixa capacidade de condução térmica, então o conjunto é inserido nos tubos ou canais por onde o fluido escoará (Elwenspoek, 1999).

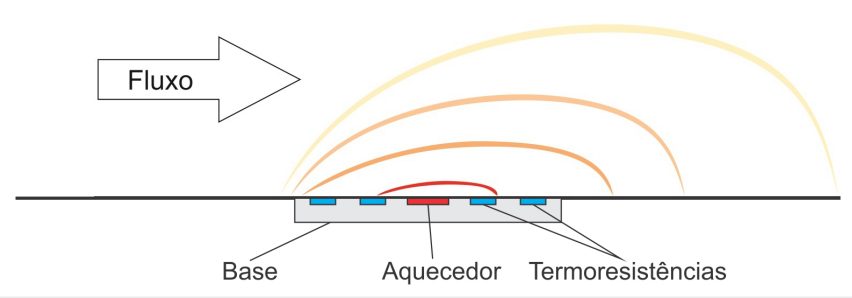

Figura 2. Sensor de fluxo do tipo Calorimétrico.

O sinal produzido por um sensor calorimétrico depende unicamente do comportamento do campo térmico gerado no fluido pelo elemento aquecedor. A convecção faz com que o calor se propague naturalmente pelo fluido de forma ascendente, enquanto o fluido se encarrega de transportar parte da energia térmica na mesma direção de seu movimento. Logo, na ausência de fluxo os elementos sensores receberão a mesma quantidade de energia térmica devido à sua simetria em relação ao aquecedor, consequentemente a variação das resistências em função da temperatura será uniforme. Ao escoar o fluido através da região sob influência do aquecedor, o campo térmico sofrerá distorções e, por conseguinte, os elementos sensores apresentarão resistências diferentes (Sazhin, 2013). Desta forma, podese estimar a velocidade do fluido em função da diferença de temperatura das termorresistências.

Os chamados sensores TOF (time of flight ou tempo de voo) recebem esse nome devido ao seu princípio de funcionamento. Trata-se de uma operação dinâmica, onde um pulso térmico é emitido pelo aquecedor e captado pelo elemento sensor após algum tempo, como demonstrado na Figura 3. O tempo compreendido desde a emissão do pulso até que o mesmo sensibilize o termistor depende unicamente da velocidade do fluido, independente de qual seja ele ou em quais condições se encontra (Sazhin, 2013). 


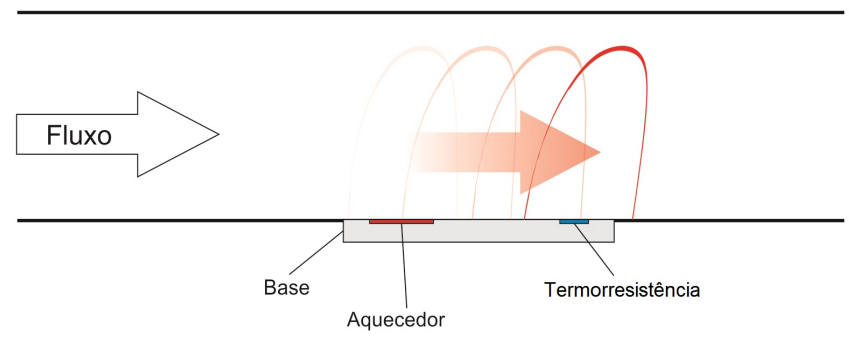

Figura 3. Sensor de fluxo do tipo Time of Flight.

A utilização de microssensores de fluxo de massa térmica tem se tornado cada vez mais difundida nos campos da robótica, aeronáutica, medicina, entre outros (Haasl e Stemme, 2008). Sazhin (2013), por exemplo, apresenta o uso desse tipo de sensores em sistemas automobilísticos, mais especificamente nos mecanismos de injeção eletrônica e no controle da mistura ar-combustível que entra no motor.

Outras aplicações bastante promissoras estão concentradas na área médica. $\mathrm{O}$ controle respiratório pode ser feito utilizando dispositivos de características muito peculiares instalados nas vias do paciente, que devem levar em consideração a direção do fluido, além de suportar aspectos complicadores como a variação de temperatura e a possibilidade de que partículas se depositem sobre o sensor, conforme mostrado por Haasl e Stemme (2008). Alguns sensores são pequenos o bastante para serem inseridos em um cateter e utilizados no monitoramento e controle da pressão arterial de pacientes em estado crítico ou mesmo para aplicações de pequenas doses de alguns tipos de drogas e medicamentos.

Wang et al. (2018) apresentam a implementação de um microssensor do tipo hot-wire dentro de um encapsulamento de circuito integrado, visando sua utilização no contexto de alta potência. No âmbito da Internet das Coisas (IoT), $\mathrm{Xu}$ et al. (2018) lançaram mão de recursos da tecnologia MEMS e da CMOS para desenvolver o protótipo de um módulo sensor wireless com intuito de ser utilizado como nó de redes de sensores.

O controle de velocidade de veículos aéreos, tripulados ou não, é outro dos possíveis usos já vislumbrados para os microssensores de fluxo concebidos sob a filosofia MEMS. Para esse fim, os sensores são sempre instalados na fuselagem e, desta forma, a velocidade do veículo é mensurada com base no fluxo de ar que escoa em torno do mesmo (Tokutake et al., 2011; ller et al., 2012).

\section{METODOLOGIA}

O desenvolvimento do foi realizado em ambiente computacional, por meio do software COMSOL Multiphysics ${ }^{\circledR}$. Esta tarefa foi executada seguindo as quatro etapas, a seguir:

- definição da geometria,. Verificou-se o quanto a disposição e dimensões dos elementos impactam no sinal de saída do microssensor;

- aplicação de materiais. Analisou-se as propriedades físicas de alguns metais e semicondutores. Escolheu- se os materiais de acordo com a finalidade de cada elemento do dispositivo;

- caracterização da física do processo. Consistiu-se na escolha do fluido que passaria pelo sensor, sua velocidade temperatura e regime de escoamento. Nessa etapa também caracterizaram-se os fenômenos eletromagnéticos relacionados ao funcionamento do dispositivo;

- testes e simulações. Simulou-se a presença de um fluxo de ar variável passando pelo microssensor, emperatura dos elementos e do fluido, bem como a resistência e o sinal de saída.

$\mathrm{Na}$ forma final do microssensor optou-se por uma base de silício monocristalino, escolhido devido sua alta rigidez dielétrica, com dimensões de 2,4 x $6 \mathrm{~mm}$ e $150 \mu \mathrm{m}$ de espessura. Centralizado sobre a base situa-se o elemento aquecedor feito de níquel, material capaz de dissipar um potência considerável em forma de energia térmica e cujas propriedades elétricas e térmicas são exibidas na Tabela 1.

Tabela 1. Propriedades físicas do níquel.

\begin{tabular}{cc} 
Propriedade & Valor \\
\hline Resistividade a $20^{\circ} \mathrm{C}\left(\rho_{0}\right)$ & $6,9 \times 10^{-8} \Omega \mathrm{m}$ \\
Expansão térmica $(\alpha)$ & $13,4 \times 10^{-6} \mathrm{~K}^{-1}$ \\
Capacidade calorífica $(\mathcal{C})$ & $0,44 \mathrm{~J} \mathrm{~g}^{-1} \mathrm{~K}^{-1}$ \\
Condutividade elétrica $(\sigma)$ & $10,4 \times 10^{6} \mathrm{~S} \mathrm{~m}^{-1}$ \\
Condutividade térmica $(\kappa)$ & $90,7 \mathrm{~W} \mathrm{~m}^{-1} \mathrm{~K}^{-1}$ \\
\hline
\end{tabular}

Finalmente, alocaram-se quatro RTDs (Resistence Temperature Dependent) simetricamente em relação ao elemento aquecedor. Os RTDs possuem 1,8 mm de comprimento, 50 $\mu \mathrm{m}$ de largura e $10 \mu \mathrm{m}$ de espessura. Escolheu-se a platina como elemento metal para os RTDs uma vez que esta não apresenta condutividade elétrica e térmica se comparada a outros metais. Além disto, a platina varia sua resistência em função da temperatura de forma aproximadamente linear e possui baixo coeficiente de expansão térmica se comparado a outros metais, o que evita deformação mecânica do dispositivo. Outra vantagem é a capacidade calorífica relativamente baixa, levando a uma menor dissipação de energia térmica em função da energia elétrica que alimenta a ponte. Na Tabela 2 são demonstrados os valores característicos da platina.

Tabela 2. Propriedades físicas da platina.

\begin{tabular}{cc} 
Propriedade & Unidade \\
\hline Resistividade a $20^{\circ} \mathrm{C}\left(\rho_{0}\right)$ & $10,6 \times 10^{-8} \Omega \mathrm{m}$ \\
Expansão térmica $(\alpha)$ & $8,8 \times 10^{-6} \mathrm{~K}^{-1}$ \\
Capacidade calorífica $(\mathcal{C})$ & $0,13 \mathrm{~J} \mathrm{~g}^{-1} \mathrm{~K}^{-1}$ \\
Condutividade elétrica $(\sigma)$ & $9,4 \times 10^{6} \mathrm{~S} \mathrm{~m}^{-1}$ \\
Condutividade térmica $(\kappa)$ & $71,6 \mathrm{~W} \mathrm{~m}^{-1} \mathrm{~K}^{-1}$ \\
\hline
\end{tabular}

Na Figura 4, os aspectos construtivos do dispositivo são apresentados em sua forma final.

O circuito elétrico do sensor é formado pelo aquecedor e pelos RTDs. Nos terminais do elemento aquecedor, aplicouse uma diferença de potencial de $3,3 \mathrm{~V}$, que produziu uma corrente e, consequentemente, houve dissipação de energia térmica pelo efeito Joule. Os RTDs, por sua vez, estão configurados de modo a formar uma Ponte de Wheatstone, conforme mostra a Figura 5, sendo que a ponte foi alimentada por $10 \mathrm{mV}$, a fim de que a energia térmica dissipada nesses elementos pudesse ser desprezível e a temperatura 


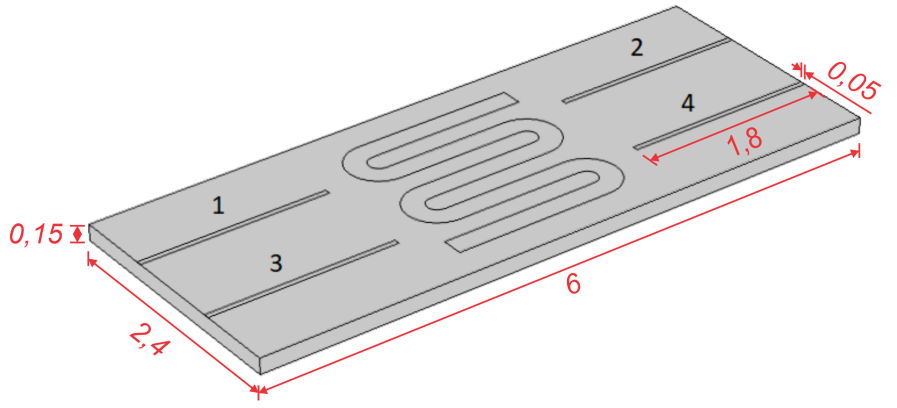

Figura 4. Microssensor de fluxo de massa projetado. Sobre a base encontram-se o elemento aquecedor (ao centro) e os RTDs, numerados de 1 a 4 . Dimensões em milímetros.

desses elementos dependessem majoritariamente do calor transportado pelo fluido.
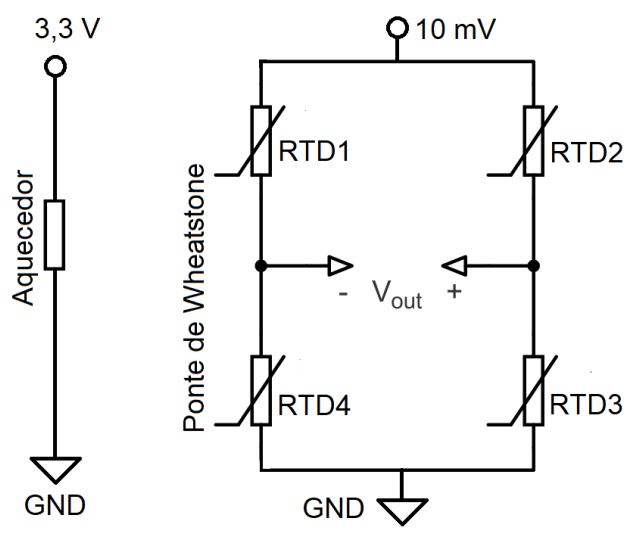

Figura 5. Representação elétrica do microssensor. Elemento aquecedor à esquerda e, à direita, o arranjo dos elementos sensores.

A resistência de cada RTD é calculada por meio da Equação 1.

$$
R=R_{0}\left(1+\alpha\left(T-T_{0}\right)\right) .
$$

em que $T_{0}$ é a temperatura de referência $\left(20^{\circ} \mathrm{C}\right), R_{0}$ é a resistência de cada RTD nesta mesma temperatura $(35,2$ $\Omega$ ). Finalmente, $\alpha$ é o coeficiente de expansão térmica da platina $\left(8,8 \times 10^{-6} K^{-1}\right)$.

A tensão de saída do microssensor $\left(V_{\text {out }}\right)$ é aproximada pela Equação 2.

$$
V_{\text {out }}=V_{\text {in }}\left(\frac{R_{3}}{R_{1}+R_{3}}-\frac{R_{4}}{R_{2}+R_{4}}\right) .
$$

Por fim, realizou-se um estudo que consistiu em simular a aplicação de um fluxo de ar, com temperatura inicial de $20^{\circ} \mathrm{C}$, e coletar a tensão de resposta nos terminais do dispositivo. Para este teste, variou-se a velocidade do ar gradativamente de 0 a $18 \mathrm{~m} / \mathrm{s}$ num canal de seção transversal de $5,76 \mathrm{~mm}^{2}$. Por meio da simulação obtevese as resistências e temperaturas instantâneas de cada um dos RTDs, bem como a tensão de resposta.

\section{RESULTADOS E DISCUSSÕES}

Por meio da simulação dinâmica do microssensor de fluxo de massa térmica, em que o mesmo foi submetido à passagem de ar em diferentes velocidades, percebeu-se a não uniformidade do campo térmico produzido no interior do fluido, conforme preconizado pela teoria. A distribuição do calor deu-se em função da transferência ocasionada pelos fenômenos de condução e convecção. Na Figura 6, é mostrada a distribuição de energia térmica no interior do fluido e na superfície do dispositivo.

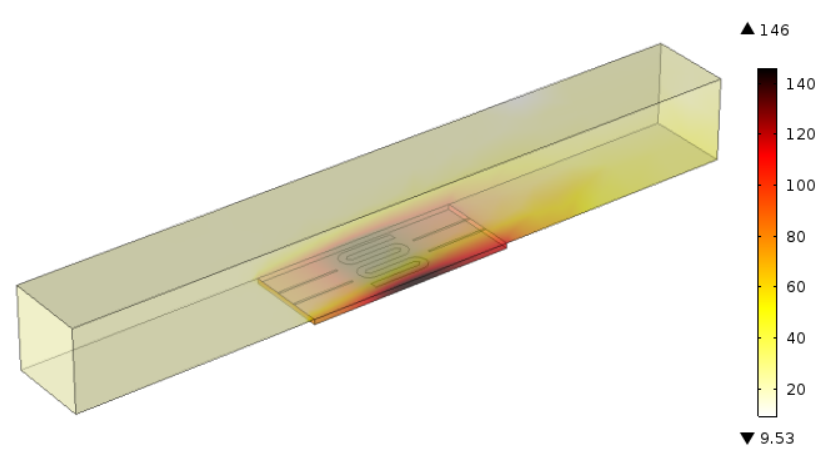

Figura 6. Distribuição da temperatura no fluido. A escala representa a temperatura $\mathrm{em}{ }^{\circ} \mathrm{C}$.

Devido à interação com o fluido em movimento, os RTDs assumiram temperaturas diferentes entre si. Por conseguinte, a resistência elétrica de cada um desses elementos sensores também variou de maneira proporcional à taxa de deslocamento do fluido ao qual o sensor está imerso e em função da posição onde os mesmos se encontravam. Os RTDs situados próximos à entrada do canal de ar (upstreams) apresentaram menor diferença de temperatura para a referência $\left(20^{\circ} \mathrm{C}\right)$ que os elementos posicionados mais próximo à saida do canal (downstream). Na Figura 7, é possível verificar a diferença de temperaturas entre os elementos sensores dos pares 1-2 e 3-4.

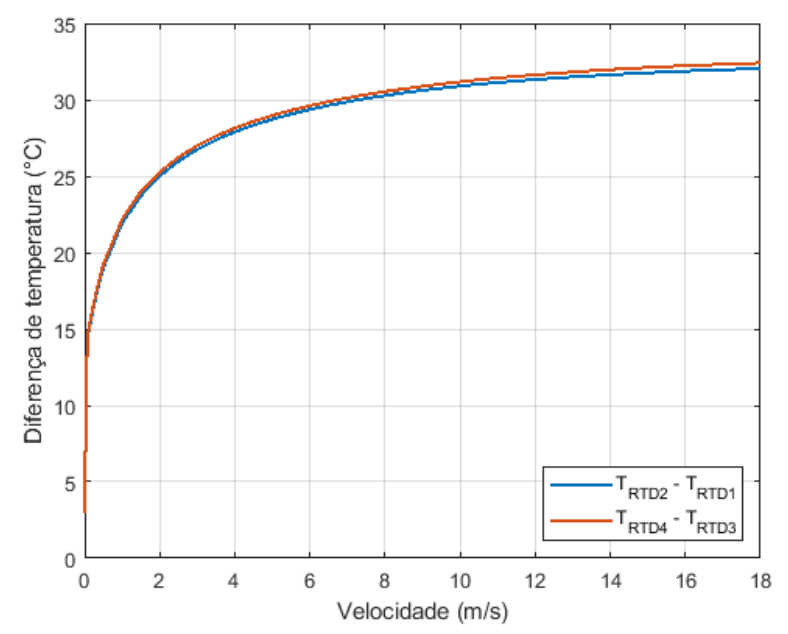

Figura 7. Diferença de temperatura entre um RTD e seus respectivos pares.

O comportamento da temperatura na superfície do sensor reflete diretamente nos RTDs. Uma vez que o coeficiente de 
expansão térmica da platina é positivo, os elementos que possuíam temperaturas maiores também assumiam valores de resistência superiores. Na Figura 8 são mostrados os valores de resistência assumidos por cada elemento sensor em função da velocidade do ar que passava pelo canal e interagia com a superfície do instrumento.

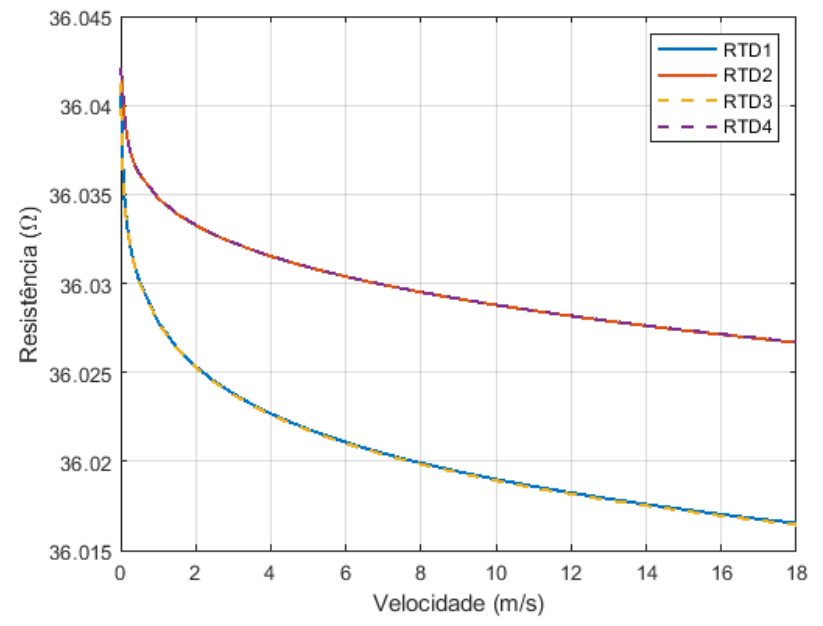

Figura 8. Variação da resistência dos RTDs ao longo da aplicação do fluxo de ar.

Finalmente, na Figura 9 é mostrado o sinal obtido ao simular o fluxo de ar passando pelo sensor e incrementálo gradativamente. Nota-se que o sinal começa a saturar a partir dos $14 \mathrm{~m} / \mathrm{s}$, portanto conclui-se que até esta velocidade o aparato pode ser utilizado como instrumento de medição.

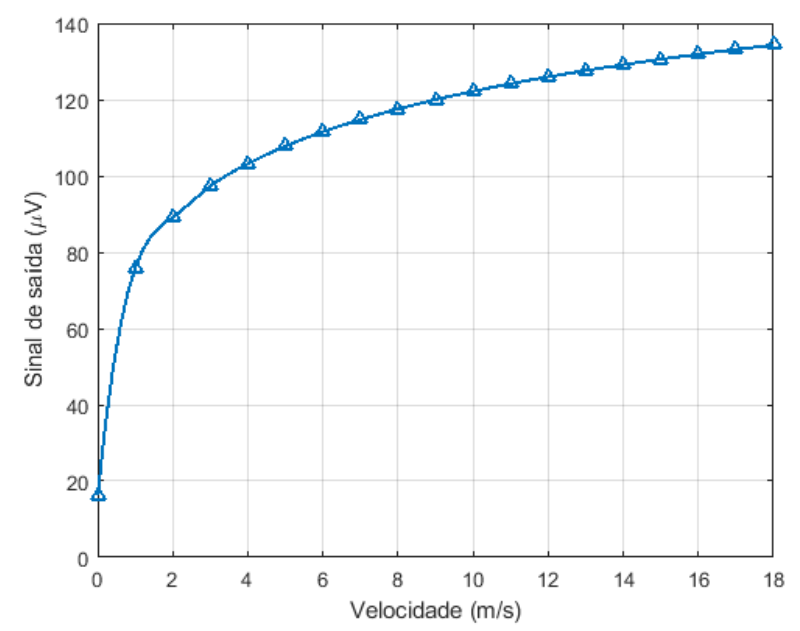

Figura 9. Sinal de tensão na saída do microssensor quando aplicado diferentes valores de vazão.

\section{CONCLUSÕES}

O microssensor de fluxo de massa do tipo calorimétrico foi desenvolvido com base em princípios termodinâmicos e eletromagnéticos, a saber, a transferência térmica em sólidos e fluidos e da dissipação de energia através do efeito Joule. Os materiais utilizados foram escolhidos com base nas propriedades físicas dos mesmos, buscando maior eficiência no funcionamento do dispositivo.

Por meio das simulações verificou-se que o sensor é adequado para operar na faixa de velocidade entre 0 e 14 $\mathrm{m} / \mathrm{s}$. A faixa de medição atende alguns instrumentos desta categoria. Além disto, as dimensões do aparato propiciam sua aplicação em uma vasta gama de sistemas, seja em eletrônica embarcada, redes sensoriais, aplicações industriais, etc.

O estudo de outros materiais e componentes, e manipulações nas características construtivas (geometria, dimensões e disposição dos RTDs) também pode implicar num ganho de sensibilidade, permitindo assim ampliar o range de saída do sensor. Pretende-se, em trabalhos futuros, construir um protótipo físico do aparato simulado com o objetivo de validar os resultados computacionais, bem como sua aplicação e viabilidade em sistemas reais.

\section{AGRADECIMENTOS}

Os autores agradecem à Fapemig (Fundação de Amparo à Pesquisa do Estado de Minas Gerais), ao Engenheiro Lucas Ribeiro, da Embraer e aos colaboradores do Centro Universitário de Sete Lagoas.

\section{REFERÊNCIAS}

Barmpakos, D., Famelis, I., Moschos, A., Marinatos, D., e Kaltsas, G. (2019). Design and evaluation of a multidirectional thermal flow sensor on flexible substrate. Journal of Sensors, 2019, 1-10. doi:10.1155/2019/8476489.

Djuzhev, N.A., Novikov, D.V., Demin, G.D., Ovodov, A.I., e Ryabov, V.T. (2018). An experimental study on mems-based gas flow sensor for wide range flow measurements. In 2018 IEEE Sensors Applications Symposium (SAS), 1-4. doi:10.1109/SAS.2018.8336727.

Elwenspoek, M. (1999). Thermal flow micro sensors. In International Semiconductor Conference. IEEE. doi: 10.1109/smicnd.1999.810580.

Gerdroodbary, M.B., Anazadehsayed, A., Hassanvand, A., e Moradi, R. (2018). Calibration of low-pressure mems gas sensor for detection of hydrogen gas. International Journal of Hydrogen Energy, 43(11), 5770-5782.

Haasl, S. e Stemme, G. (2008). Flow sensors. In Comprehensive Microsystems, 209-272. Elsevier. doi:10. 1016/b978-044452190-3.00054-9.

Inoue, R., Nogami, H., Higurashi, E., e Sawada, R. (2018). Simultaneous measurement of a blood flow and a contact pressure. In 11th International Conference on BioInspired Systems and Signal Processing, BIOSIGNALS 2018-Part of 11th International Joint Conference on Biomedical Engineering Systems and Technologies, BIOSTEC 2018, 48-53. SciTePress.

ller, J.M., Paul, O., e Burgard, W. (2012). Probabilistic velocity estimation for autonomous miniature airships using thermal air flow sensors. IEEE International Conference on Robotics \& Automation, 39-44.

Lu, Y. (2017). Industry 4.0: A survey on technologies, applications and open research issues. Journal of Industrial Information Integration, 6, 1 - 10. doi:https://doi. org/10.1016/j.jii.2017.04.005.

Maluf, N. (2002). An introduction to microelectromechanical systems engineering. Measurement Science and 
Technology, 13, 229-229. doi:10.1088/0957-0233/13/2/ 701.

Melnyk, M., Rubacha, J., Kamisinski, T., e Majchrzak, A. (2018). Application of mems sensors for the automation of a laboratory stand for the measurement of the flow resistance of porous materials. In $2018 \mathrm{XIV}$ th International Conference on Perspective Technologies and Methods in MEMS Design (MEMSTECH), 28-34. doi:10.1109/MEMSTECH.2018.8365695.

Sazhin, O. (2013). Novel mass air flow meter for automobile industry based on thermal flow microsensor i. analytical model and microsensor. Flow Measurement and Instrumentation, 30, 60-65.

Tokutake, H., Sunada, S., e Fujinaga, J. (2011). Attitude control of a small uav using a flow sensor system. Journal of System Design and Dynamics, 5, 16.

Vaidya, S., Ambad, P., e Bhosle, S. (2018). Industry 4.0 - a glimpse. Procedia Manufacturing, 20, 233 - 238. doi:https://doi.org/10.1016/j.promfg.2018.02.034. 2nd International Conference on Materials, Manufacturing and Design Engineering (iCMMD2017), 11-12 December 2017, MIT Aurangabad, Maharashtra, INDIA.

Wang, T., Wang, J., He, J., Wu, C., Luo, W., Shuai, Y., Zhang, W., Chen, X., Zhang, J., e Lin, J. (2018). A comprehensive study of a micro-channel heat sink using integrated thin-film temperature sensors. Sensors, 18(1), 299.

Xu, W., Lijin, B., Duan, M., Wang, X., Wicaksana, J., Min, A., Ahmed, M., Wang, R., Fang, N.X., Bermak, A., e Lee, Y. (2018). A wireless dual-mode micro thermal flow sensor system with extended flow range by using cmos-mems process. In 2018 IEEE Micro Electro Mechanical Systems (MEMS), 824-827. doi:10.1109/ MEMSYS.2018.8346682. 